\title{
On-Surface Synthesis with Atomic Hydrogen
}

\author{
Rafal Zuzak, Andrej Jančařík, Andre Gourdon, Marek Szymonski, and Szymon Godlewski*
}

Cite This: ACS Nano 2020, 14, 13316-13323

Read Online

ABSTRACT: Surface-assisted synthesis has become a powerful approach for generation of molecular nanostructures, which could not be obtained via traditional solution chemistry. Nowadays there is an intensive search for reactions that could proceed on flat surfaces in order to boost the versatility and applicability of synthesized nanoobjects. Here we propose application of atomic hydrogen combined with on-surface synthesis in order to tune the reaction pathways. We demonstrate that atomic hydrogen could be widely applied: (1) as a cleaning tool, which allows removal of halogen residues from the surface after Ullmann couplings/polymerization, (2) by reaction with surface organometallics to provide stable hydrogenated species, and (3)

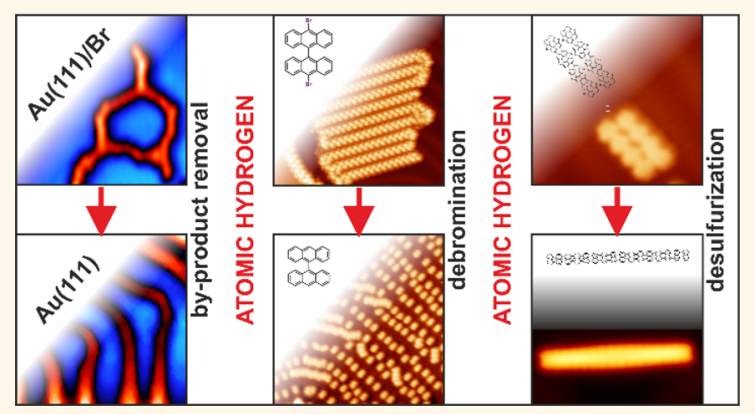
as a reagent for debromination or desulfurization of adsorbed species.

KEYWORDS: on-surface synthesis, graphene nanoribbon, hydrogenation, atomic hydrogen, molecular polymers, organometallic state

S ince its introduction at the turn of the 21 st century, the on-surface synthesis approach has proven to be extremely successful in providing functional nanomaterials, whose structure could be controlled down to even single atoms. ${ }^{1}$ Over the past several years, the surface-assisted nanomaterial synthesis has attracted tremendous researcher attention as an alternative to conventional solution chemistry, being particularly effective in the generation of polymers, ${ }^{2}$ graphene nanoribbons, ${ }^{3,4}$ nanoflakes, ${ }^{5-8}$ or elusive single molecules. $^{9-11}$ The approach enables precise synthesis of molecular nanoarchitectures exhibiting the desired structural, chemical, electronic, and magnetic properties ${ }^{11}$ using custom designed molecular precursors. Continuous development of the available on-surface synthesis toolkits allows the generation of more sophisticated and complex molecular nanodevices. Further development of the on-surface synthesis approach would depend on the introduction of concepts giving the possibility of increasing the control of reactions at the atomic level, which at the same time will allow the production of nanostructures with given specific functionalities. In this context, approaches and reagents allowing to further transform on-surface nanoarchitectures are indispensable.

On-surface synthesis of extended molecular nanostructures is commonly achieved by aryl-aryl coupling following an Ullmann-like reaction scheme on appropriately chosen metallic surfaces playing a catalytic role in polymerization. ${ }^{1,2,12,13}$ Most often, custom-designed molecular precursors are equipped with halogen atoms as leaving groups on the desired active sites. As a result, when the polymerization step is activated, halogens remain as the major byproduct contaminating the surface and hence influencing the overall reaction process. ${ }^{14-16}$
While halogen atoms exposed at the surface might be removed by higher temperature annealing, which is usually associated with cyclodehydrogenation leading to planarization of previously generated polymers, ${ }^{3,4}$ removal of surface halogens is still a challenging problem if higher temperature annealing is not needed or especially impossible to carry out. Simultaneously, there is an intensive search for alternative surfaceassisted reaction pathways that would improve the abilities to control, specify, and tune the functionalities of molecular systems and extend the versatility of the approach. ${ }^{1}$

Here we demonstrate an approach based on application of atomic hydrogen in on-surface synthesis. From our experiments, we show that residual bromine as a byproduct of surface-assisted polymerization could be efficiently removed from the surface by appropriate reaction with atomic hydrogen leaving the generated surface polymers intact. Furthermore, the concept of molecular nanostructures modification based on atomic hydrogen is introduced. We indicate that surfacegenerated covalent architectures coupled through organometallic chemistry may be efficiently separated by our concept. This shows that the approach provides not only a versatile toolkit for generation of individual, not cross-coupled, covalent nanostructures but also enables atomic saturation of GNRs termination. Finally, with properly chosen molecular pre-

Received: June 22, 2020

Accepted: September 8, 2020

Published: September 8, 2020 


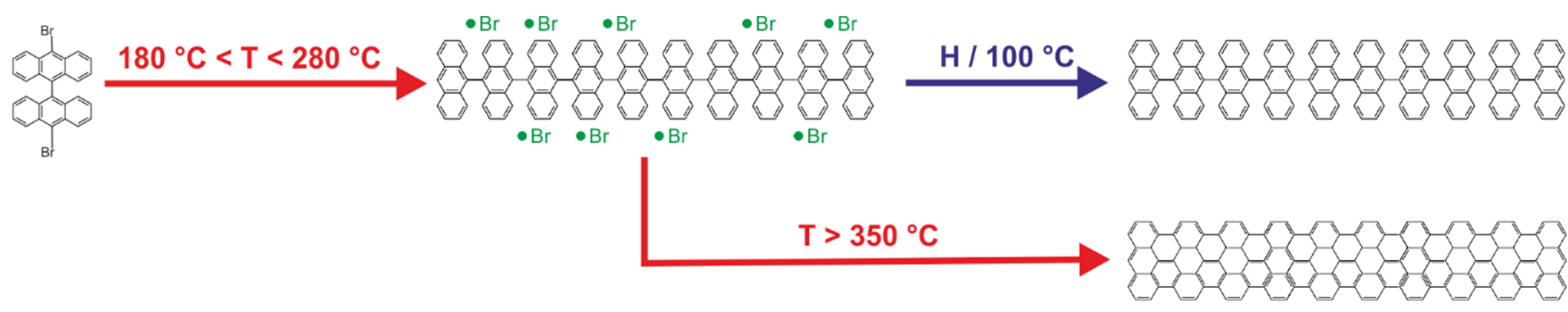

Figure 1. Surface cleaning conditions to get a polyanthryl polymer. The first step, an Ullmann-like coupling of DBBA on a metallic surface, yields polyanthryl polymers surrounded by adsorbed bromine atoms. Thermal desorption of these atoms can be done above $280{ }^{\circ} \mathrm{C}$, but at these temperatures, intramolecular cyclodehydrogenations also proceeds. In contrast, treatment by atomic hydrogen at $100{ }^{\circ} \mathrm{C}$ only removes the adsorbed bromine atoms, leaving intact the polymers.
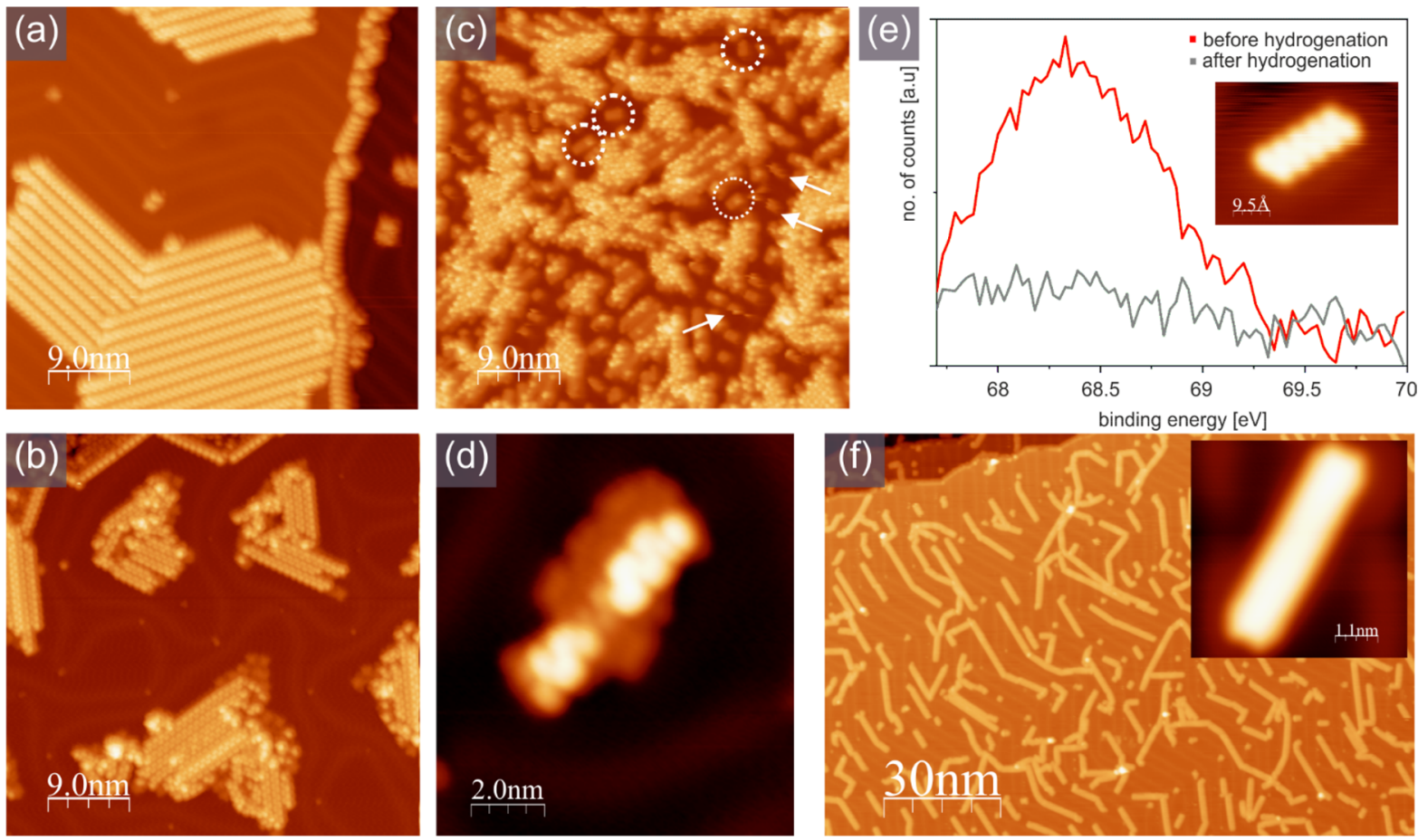

Figure 2. Hydrogen treatment of DBBA precursors: (a) STM image of self-assembled DBBA molecules; (b) DBBA polymers with surrounding $\mathrm{Br}$ atoms obtained after annealing at $180{ }^{\circ} \mathrm{C}$; (c) STM image of the sample after atomic hydrogen treatment at $100{ }^{\circ} \mathrm{C}-$ the flexible DBBA precursors are found within islands, white dashed circles mark exemplary isolated polymers (magnified image is shown as inset in (e)); white arrows highlight the mobile DBBA polymers; (d) high-resolution STM image of the small island comprised of DBBA polymers after atomic hydrogen treatment at $100{ }^{\circ} \mathrm{C}$ - the image clearly shows that the assembly is not planar and contains polymers placed on top of the first layer polymers; (e) XPS data obtained for Br 3d resonance before (red) and after (gray) application of atomic hydrogen flux at $100{ }^{\circ} \mathrm{C}$, disappearance of the resonance resembles effective removal of $\mathrm{Br}$ by atomic hydrogen flux (the inset shows high resolution STM image of a single DBBA polymer $(0.5 \mathrm{~V}, 25 \mathrm{pA})$ ); (f) large-scale STM image of 5-AGNRs generated by annealing at $350{ }^{\circ} \mathrm{C}$ the sample with DBBA polymers after application of the atomic hydrogen treatment. Scan parameters: $-1 \mathrm{~V}, 50 \mathrm{pA}(\mathrm{a}, \mathrm{b}, \mathrm{f}),-1 \mathrm{~V}, 15 \mathrm{pA}(\mathrm{c}, \mathrm{d}, \mathrm{e})$.

cursors we demonstrate that different side substituents might be exchanged when atomic hydrogen is associated with appropriate thermal treatment. This provides further insight into on-surface synthesis and gives perspectives for future developments. For the experiments we have chosen commercially available $10,10^{\prime}$-dibromo-9,9'-bianthryl (DBBA) precursors and 3,4,8,9-tetrabromoperyleno[1,12$\left.b c d: 6,7-b^{\prime} c^{\prime} d^{\prime}\right]$ dithiophene (TBPDT) molecules synthesized following the recipe described by Zagryanyarski and coworkers. $^{22}$ The experiments have been conducted on the $\mathrm{Au}(111)$ monocrystal.

\section{RESULTS AND DISCUSSION}

Removal of Residual Bromine Atoms. The on-surface path toward extended molecular nanoarchitectures most often relies on aryl-aryl coupling within the Ullmann-like reaction scheme with the metallic substrate providing catalytic activity. ${ }^{1,2,12-14}$ The usual design approach is based on precursors equipped with halogen atoms being protectors of the prepared active sites. As a result, when the polymerization step is activated, halogens remain as the major byproduct contaminating the surface and hence influencing the overall reaction process. ${ }^{15-17}$ While bromine adsorbed on a surface may influence the reaction processes, e.g., the kinetics, it also modifies the substrate surface structure, removing the so-called herringbone pattern, and lifts the work function. ${ }^{23}$ In effect, it does not allow the study of polymers or other nanostructures (e.g., 2-dimensional covalent molecular lattices) as they are unaffected. While halogen atoms exposed at the surface might be removed by higher temperature annealing, this procedure 
can lead to unwanted reactions like cyclodehydrogenation, lateral fusion of polymers, or decomposition, so that this type of cleaning of surfaces is still a challenging problem. As a prototype example, we have explored the well-known route to synthesize 7-AGNR from the DBBA precursor according to ref 3. The experimental procedure leading to polyanthryl polymers (Figure 1) comprises deposition of DBBA on a metallic surface in UHV (Figure 2a), followed by annealing at 110-200 ${ }^{\circ} \mathrm{C},{ }^{18-22}$ which initiates Ullmann-like surface coupling. ${ }^{2}$ In such a case, the linear polymers are surrounded by bromine atoms, the reaction byproduct, which cannot be efficiently removed below $200{ }^{\circ} \mathrm{C}$ (Figure $2 \mathrm{~b}$ ). Increasing the annealing temperature to $280{ }^{\circ} \mathrm{C}$ induces bromine atom desorption but also initiates polymer transformation; i.e., under such conditions, we observe emergence of the intramolecular cyclodehydrogenation onset, which leads to planarization yielding graphene nanoribbons ${ }^{3,4}$ or nanoflakes. ${ }^{2}$ Whereas it was proposed to apply molecular hydrogen to remove bromine byproducts, ${ }^{24}$ we have found this approach to be inefficient, especially in cases where the molecular nanoarchitectures are tightly surrounded by bromine or even when bromine is incorporated between the molecular nanostructures as in the case of DBBA polymers (Figure 2b). In contrast, we have applied atomic hydrogen flux, stabilized at the gas pressure of 1 $\times 10^{-7}$ mbar, and kept the surface at a slightly elevated temperature, i.e., at $100{ }^{\circ} \mathrm{C}$ lasting for $10-20 \mathrm{~min}$. This procedure leads to very efficient bromine removal while keeping the polymers unaffected, which is demonstrated in Figure $2 c-f$. After application of the atomic hydrogen procedure, highly nonplanar and flexible DBBA polymer chains might still be under the influence of interchain forces, e.g., $\pi-\pi$ stacking, resulting in their gathering into not wellordered assemblies. This makes their direct differentiation based on STM appearance difficult, as clearly illustrated in Figure $2 \mathrm{c}$,d. However, as indicated by white dashed circles in Figure 2c, isolated DBBA polymers could also be found on $\mathrm{Au}(111)$ and subsequently visualized by high-resolution STM imaging as indicated by the inset in Figure 2e. Furthermore, the above-described assembly of DBBA polymers into islands does not allow us to visualize with STM the disappearance of $\mathrm{Br}$ atoms, which initially-before atomic hydrogen treatment-surround the DBBA polymers. Therefore, in order to trace and doubtlessly prove the $\mathrm{Br}$ removal, we have performed additional in situ X-ray photoelectron spectroscopy (XPS) measurements. This is demonstrated in Figure 2e presenting the $\mathrm{Br} 3 \mathrm{~d}$ resonance before and after the hydrogen treatment, while for the sample with on-surface generated DBBA polymers we can clearly notice presence of the resonance centered at approximately $68.3 \mathrm{eV}$ and after hydrogen treatment the signal is completely suppressed. This corresponds to effective $\mathrm{Br}$ removal from the surface. Further, in order to demonstrate resistance of polymers against atomic hydrogen, generation of defect-free 7-AGNRs is achieved by following the standard recipe of annealing above $280{ }^{\circ} \mathrm{C}$. The resulting nanoribbons are visualized in Figure $2 \mathrm{f}$. At this point it is important to emphasize that our protocol might be highly efficient for cleaning of on-surface generated molecular nanostructures, not only polymers but also extended 2dimensional covalent nanoarchitectures, which are currently attracting increasing attention worldwide.

Reaction with Surface Organometallics. In the mechanism of on-surface Ullmann coupling as shown in Figure 1, the first reaction step is an oxidative addition in which a surface metal atom gets inserted between the halide atom and the carbon atom bearing this halide. Most often, this intermediate is not stable at the reaction temperature, so that the reaction proceeds toward a nonplanar reductive elimination yielding the final carbon-carbon coupling. ${ }^{25}$ However, in some cases, this intermediate state is stable enough to be observed or even manipulated, ${ }^{26}$ for instance, on copper surfaces ${ }^{27}$ or when intermolecular steric crowding is large preventing the final $\mathrm{C}-\mathrm{C}$ coupling. In this work, we deposited TBPDT (Figure 3) on the $\mathrm{Au}(111)$ surface. This

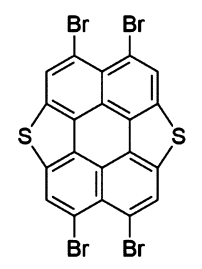

Figure 3. 4,8,9-Tetrabromoperyleno $\left[1,12-b c d: 6,7-b^{\prime} c^{\prime} d^{\prime}\right]$ dithiophene (TBPDT).

molecule was selected since it is planar, with two thiophene functions, which increases the van der Waals adsorption forces and, therefore, causes the molecule to lie flat on the surface. As the reductive elimination step is nonplanar, it was expected that this strong adsorption would render the organometallic intermediate more stable and the $\mathrm{C}-\mathrm{C}$ coupling step more energetically demanding. Furthermore, the molecule comprises four carbon-bromine bonds, which should as well stabilize the organometallic intermediate. We show here that this organometallic intermediate reacts with atomic hydrogen to give the hydrogenated molecule (Figure 4).

After deposition on the $\mathrm{Au}(111)$ surface at room temperature TBPDT molecules self-assemble into islands (Figure 5a), while the Au surface herringbone pattern is preserved. The molecular islands are transformed into organometallic wires through dehalogenation when annealed in the range of 180$280{ }^{\circ} \mathrm{C}$, as shown in Figure 5b,c. Notably, the surface herringbone pattern is strongly distorted by $\mathrm{Br}$ atoms located on the surface after precursor debromination. Within the organometallic wires, the neighboring molecules are usually connected through a pair of $\mathrm{C}-\mathrm{Au}-\mathrm{C}$ moieties, which for clarity are highlighted by violet dashed ovals in Figure $5 \mathrm{c}, \mathrm{d}$. The structures are surrounded by irregularly distributed remaining $\mathrm{Br}$ atoms indicated by a white arrow in Figure $5 \mathrm{~b}$ and white dashed circles in Figure $5 \mathrm{c}$. These $\mathrm{Br}$ atoms form also assembled chains-circles in Figure $5 b{ }^{28}$ Some of $\mathrm{Br}$ adatoms could also be found in the elbows of the distorted surface reconstruction pattern. Interestingly, these $\mathrm{Br}$ atoms significantly hamper attempts to functionalize the microscope tip with $\mathrm{CO}$ molecules due to increased interaction between $\mathrm{CO}$ and $\mathrm{Br}$. Such functionalization of the microscope tip would allow bond-resolved AFM measurements ${ }^{29}$ of the organometallic system. They are, however, difficult to achieve-in our attempts we did not manage to pick up $\mathrm{CO}$ molecules and functionalize the AFM tip, although some reports with successful imaging of the organometallic state surrounded by $\mathrm{Br}$ atoms could be found, e.g., on $\operatorname{Ag}(111) .^{30}$ Organometallic molecular stripes could be disassembled by dosing atomic $\mathrm{H}$ into the UHV chamber. Figure $5 \mathrm{~g}$ shows single molecules selfassembled into chains obtained after $5 \mathrm{~min} \mathrm{H}$ dosing at $100{ }^{\circ} \mathrm{C}$ and with gas pressure of $1 \times 10^{-7}$ mbar. The procedure removes also all surface bromine atoms as can be doubtlessly 


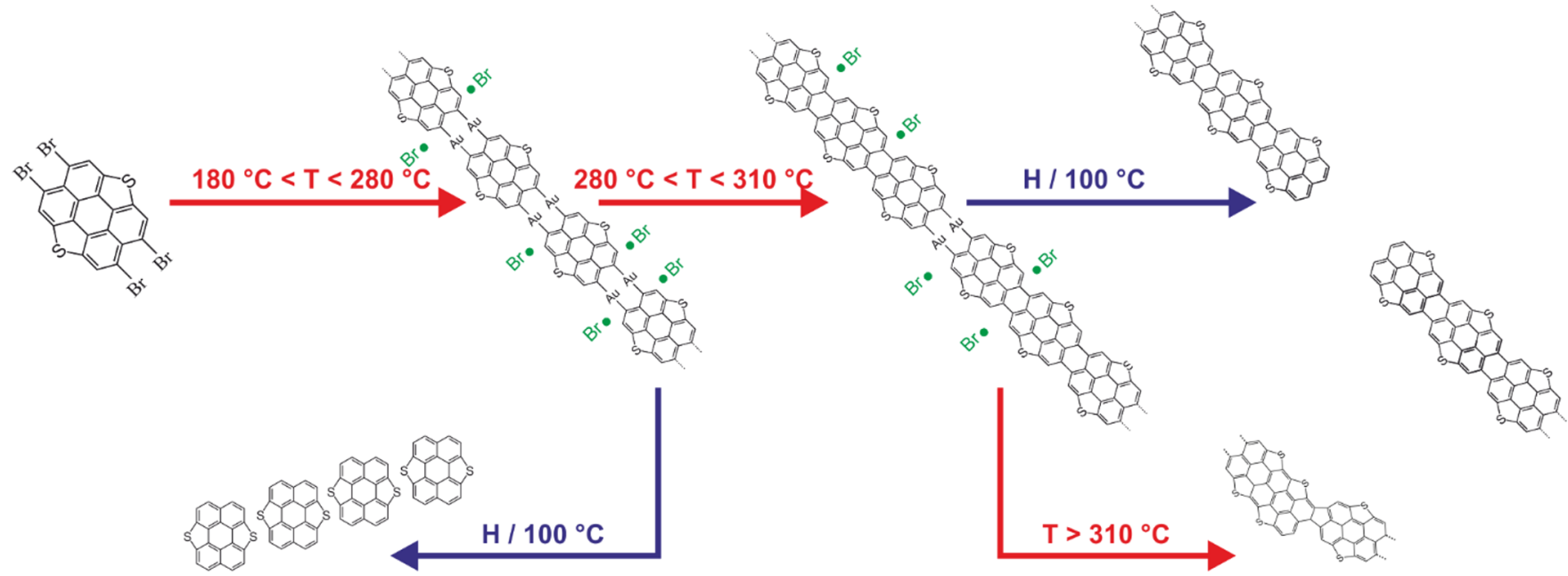

Figure 4. Annealing and atomic hydrogen treatment applied to TBPDT precursors and several possible pathways leading to organometallic structures with debrominated TBPDT molecules (top middle left), H passivation of precursors (bottom left), generation of organometallically coupled $S$ doped 5-AGNRs (top middle), H-terminated isolated S doped 5-AGNRs (right), cross-coupled 5-AGNRs (bottom, right).

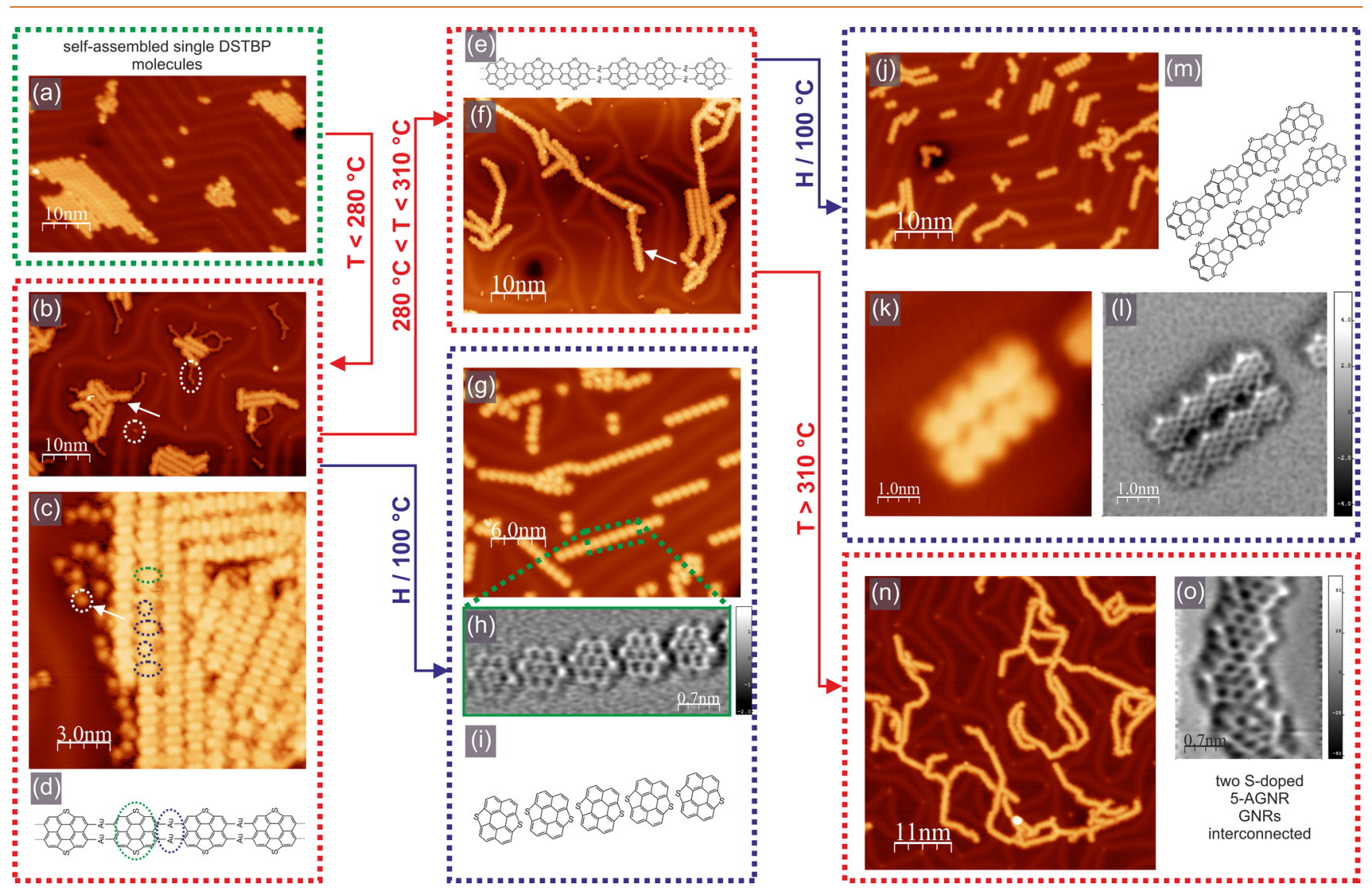

Figure 5. Combination of annealing and atomic hydrogen treatment of TBPDT precursors. (a) TBPDT self-assembled into islands after deposition at room temperature. $(b-d)$ Organometallic state consisting of debrominated TBPDT molecules; white dashed circles and ovals show bromine adsorbed on $\mathrm{Au}(111)$, while the white arrow indicates $\mathrm{Br}$ atoms surrounding organometallic units, violet ovals highlight pairs of Au atoms interconnecting neighboring molecular units, and violet circles show examples where two molecules are linked by only one Au atom; molecular units are marked by a green dashed ovals. (e, f) Organometallic state containing already some covalently linked molecular precursors, in effect S-doped 5-AGNRs, are located and interlinked within the organometallic phase; the white arrow points to bromine atoms surrounding the molecular structure even after annealing at $310^{\circ} \mathrm{C}$. $(\mathrm{g}-\mathrm{i})$ Single debrominated TBPDT molecules self-assembling into molecular stripes. (j-1) S-Doped 5-AGNRs on Au(111) after hydrogen treatment decomposing organometallic phase. (n, o) Crosslinked disordered S-doped 5-AGNRs obtained by annealing of the organometallic phase at temperatures above $310{ }^{\circ} \mathrm{C}$. Scan parameters: -1 V, 20 pA (a); +1 V, 50 pA (g); -1 V, 100 pA (b, c, f, j, k, n). 

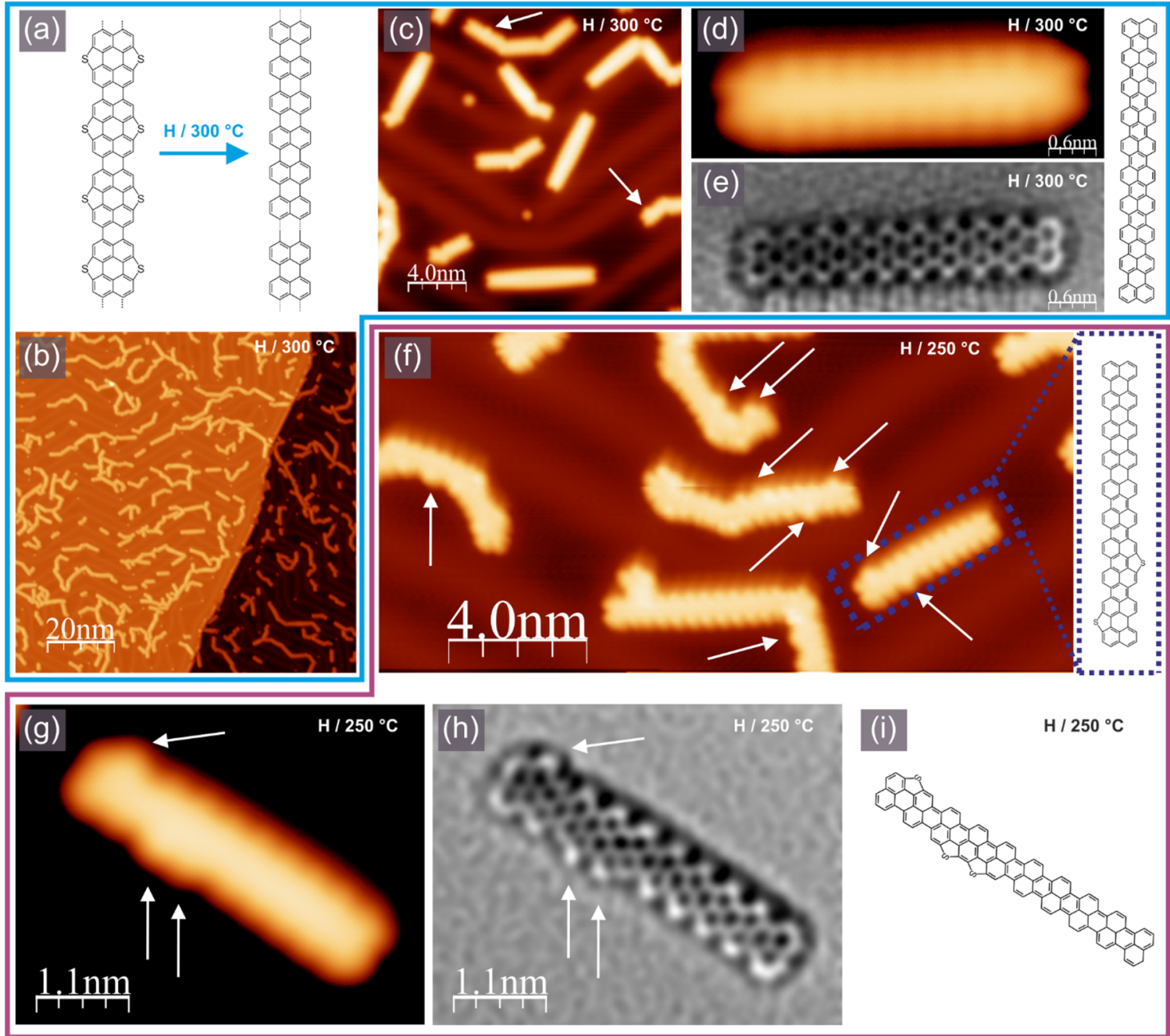

Figure 6. Removal of $S$ atoms from S-doped 5-AGNRs by atomic hydrogen flux delivered simultaneously with sample heating. (a) Schematic illustration of the process initiated at $300{ }^{\circ} \mathrm{C}$. (b) Large-scale STM image of 5-AGNRs obtained by removal of S atoms from S-doped 5AGNRs. (c) Small-scale STM image of 5-AGNRs; white arrows indicate single S atoms that remained attached to the backbone of GNRs. (d, e) STM and corresponding nc-AFM image of a perfect 5-AGNR; the structural model is shown schematically on the right-hand side. (f) High-resolution STM image with clearly visible "ladder motif" of 5-AGNRs with some single $S$ atoms still attached to the GNR backbone after $\mathrm{H}$ exposure with simultaneous heating at $250^{\circ} \mathrm{C}$, exemplary one marked with a dashed violet rectangle, structural model shown on the right. Examples of $S$ atoms are marked by white arrows within the STM topography. (g, h) High-resolution STM and corresponding nc-AFM images of a 5-AGNR with three remaining $S$ atoms still attached to the GNR backbone generated after atomic hydrogen treatment at $250{ }^{\circ} \mathrm{C}$; white arrows mark the $S$ atoms, the structural scheme of the GNR is shown in (i). Scan parameters: $-1 \mathrm{~V}, 100 \mathrm{pA}(\mathrm{b}, \mathrm{c}, \mathrm{f}),-1 \mathrm{~V}, 15 \mathrm{pA}(\mathrm{d}$, g).

inferred from STM topographies. During $\mathrm{H}$ dosing, C-Au bonds are cleaved and the precursors get passivated by $\mathrm{H}$ atoms. High-resolution bond-resolved nc-AFM imaging ${ }^{29}$ confirms self-assembly and proves that the process do not influence the side-attached $S$ atoms, as documented in Figure 5h. The experiment demonstrates also that at least annealing up to $280{ }^{\circ} \mathrm{C}$ provides no signs of dimerization or oligomerization between molecular building blocks and passivated precursors appear as self-assembled single entities. As expected, the relatively high stability of the organometallic phase, $^{31}$ quite uncommon for organometallic structures on $\mathrm{Au}(111)$, could be linked with the fact that planar neighboring molecular units are interlinked by pairs of $\mathrm{C}-\mathrm{Au}-\mathrm{C}$ motifs. This also suggests that generation of S-doped 5-AGNRs by direct fusion of neighboring precursors associated with formation of hexagonal rings might possibly be achieved only at high annealing temperatures, which carries the danger of unwanted cross-coupling between neighboring ribbons. Deliberate annealing in the range between 280 and $310{ }^{\circ} \mathrm{C}$ leads to the organometallic phase of already covalently fused precursors with newly formed $\mathrm{C}-\mathrm{C}$ bonds interlinked by $\mathrm{C}-\mathrm{Au}-\mathrm{C}$ bonds between the S-doped 5-AGNR motifs (Figure 5e) as shown in Figure 5f. Importantly, within the above-mentioned annealing temperature range, the amount of cross-linked units was still very limited giving perspectives for generation of pure S-doped 5-AGNRs. In fact it seems that at least a fraction of nonlinear units comes from faulted stacking of neighboring precursors which are shifted and this leads to formation of only one $\mathrm{C}-\mathrm{C}$ bond instead of two $\mathrm{C}-\mathrm{C}$ connections. This results in the increased flexibility of the molecule part, which finally leads to the formation of a 5-membered ring between the units when the debrominated and thus activated site attaches to the side of the neighboring unit (for details, see Figure S1). Interestingly, we note that there are remaining $\mathrm{Br}$ atoms 
located near the GNR units in Figure $5 \mathrm{f}$ as indicated by a white arrow. The identification of the structural units could be achieved after atomic $\mathrm{H}$ dosing for $5 \mathrm{~min}$ at $100{ }^{\circ} \mathrm{C}$ and at a gas pressure of $1 \times 10^{-7} \mathrm{mbar}$, which destroys all organometallic linkers and also removes unwanted on-surface adsorbed $\mathrm{Br}$ atoms. Such a procedure leads to pure S-doped 5-AGNRs (Figure 5j,k) containing even over a dozen fused precursors. These GNRs are equipped with hydrogen atom saturating end parts. The bond-resolved nc-AFM image provides undeniable evidence for successful generation of $S$ doped 5-AGNRs (structural scheme in Figure 5m) as shown in Figure 51 .

Contrary to the above-described procedure, annealing of the organometallic state at temperatures ranging above $310{ }^{\circ} \mathrm{C}$ leads to uncontrolled linking of the units and disordered phases with short cross-linked ribbon units as visualized in Figure 5n. However, a number of diagonal interconnections (Figure 5o and Figure S1) seem to arise from fault stacking of neighboring precursors, which leads to the already mentioned generation of 5-membered rings between the subunits and growth of the polycyclic core.

Desulfurization and Debromination. While in the above exploited range of surface annealing and $\mathrm{H}$ dosing the side attached $S$ atoms remain untouched, we demonstrate below that exchange reaction leading to removal of $S$ atoms could also be initiated by choosing the appropriate conditions.

Desulfurization. When the on-surface generated S-doped 5-AGNRs are subjected to atomic hydrogen, being at the same time annealed to approximately $300{ }^{\circ} \mathrm{C}(5 \mathrm{~min}$, gas pressure 1 $\times 10^{-7} \mathrm{mbar}$ ), we observe removal of $\mathrm{S}$ atoms and saturation of GNRs by $\mathrm{H}$, which leads to separated 5-AGNRs (Figure 6a). Large-scale STM topography of the sample covered by 5AGNRs is shown in Figure 6b, while Figure 6c shows a smaller scale STM image with higher resolution. The STM scan shows that on the surface there are ideal 5-AGNRs. Exemplary highresolution STM topography supplemented by the corresponding nc-AFM image taken with a $\mathrm{CO}$ functionalized tip is presented in Figure 6d,e. The high-resolution STM image is in perfect agreement with previous reports, ${ }^{32}$ and the nc-AFM topography doubtlessly confirms removal of $S$ atoms attached originally to the polycyclic backbone. We note that part of the GNRs indicates the presence of nonlinear connections, which in a significant number of cases seem to originate from fault stacking of neighboring precursors during polymerization (Figure S1) rather than from uncontrolled cross-coupling. It could also be noticed that very rarely some residual $S$ atoms may still be attached to the GNR backbone; this is marked by white arrows in Figure 6c.

However, if the temperature of annealing during atomic $\mathrm{H}$ treatment is lowered to, e.g., $250{ }^{\circ} \mathrm{C}$, more numerous $\mathrm{S}$ atoms are still attached to the 5-AGNR backbone, thus giving a chance for heterostructure generation. These $S$ atoms are well captured in the higher resolution STM image visualized in Figure 6f, where the "ladder-like" pattern of 5-AGNRs is clearly discernible and the typical pattern disorders correspond to the remaining single $S$ atoms, again marked by white arrows. Additionally, to provide the ultimate evidence for the presence of the $S$ atoms attached to the 5-AGNR backbone the bond resolved nc-AFM imaging has been applied. Parts $g$ and $h$ of Figure 6 show the high-resolution STM topography together with the nc-AFM image of the 5-AGNR with the three remaining side attached $S$ atoms indicated by white arrows. The corresponding structural scheme is displayed in Figure $6 \mathrm{i}$ for clarity. Finally, we have found that the side $\mathrm{S}$ atoms could be completely removed from the GNRs if the atomic hydrogen treatment procedure is performed at $350{ }^{\circ} \mathrm{C}$.

Debromination. Similarly, the substitution of bromine substituents by hydrogen atoms can be obtained within DBBA on $\mathrm{Au}(111)$ by atomic hydrogen treatment at $50-100{ }^{\circ} \mathrm{C}$ as shown in Figure 7. It must be emphasized that at the lower

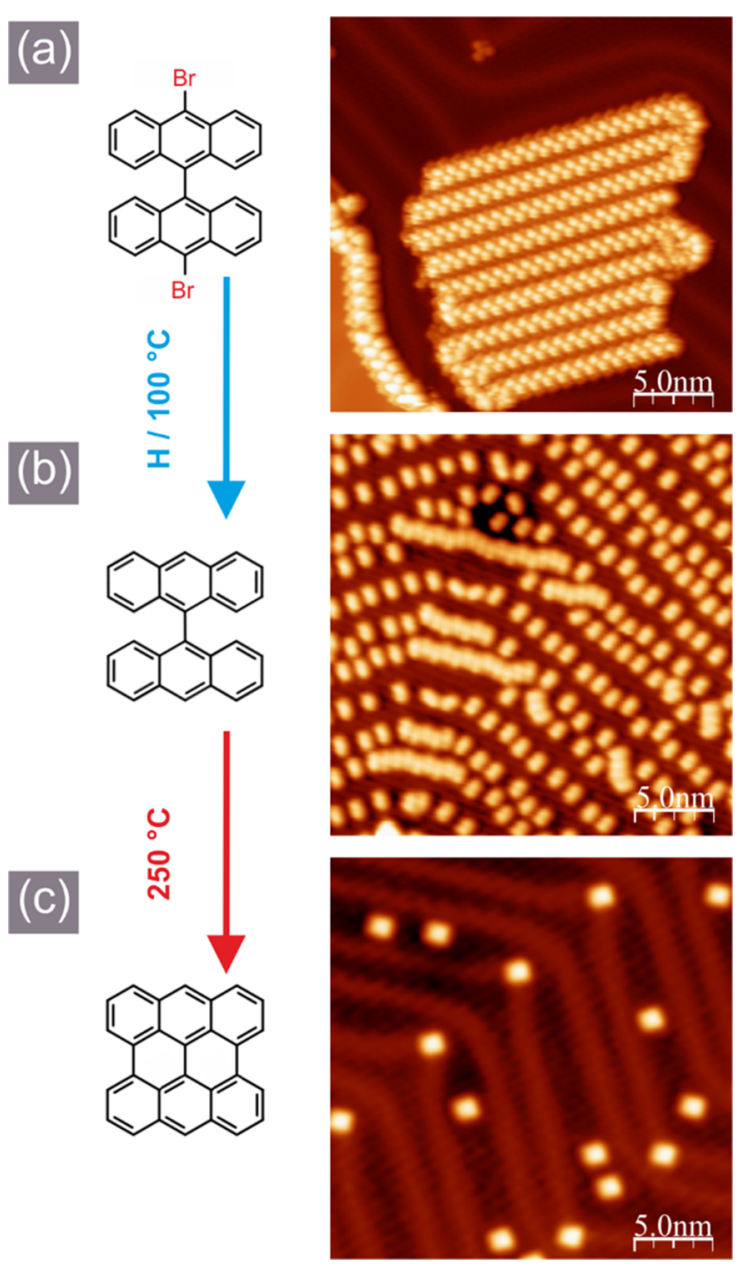

Figure 7. Passivation of DBBA precursors by atomic hydrogen and subsequent formation of bisanthene; schematic illustration of the process and STM images of self-assembled DBBA precursors (a), $9,9^{\prime}$-bianthracene generated by atomic hydrogen exposure (b), and bisanthene molecules formed by annealing associated by cyclodehydrogenation (c). Scan parameters: $-1 \mathrm{~V}, 50 \mathrm{pA}$.

limit of this temperature range DBBA is only physisorbed on the gold surface, without any prior formation of an organometallic intermediate. It has been demonstrated that the organometallic $\mathrm{Br}-\mathrm{Au}$-aryl intermediate could be found at the upper temperature limit of our hydrogenation procedure, i.e., at $100{ }^{\circ} \mathrm{C} .{ }^{31}$ Taking into account our experiments hydrogenating the precursors already at $50{ }^{\circ} \mathrm{C}$, we can conclude that the atomic hydrogen treatment leads to exchange of $\mathrm{Br}$ by $\mathrm{H}$ atoms and yields the 9,9'-bianthracene illustrated in Figure $7 \mathrm{~b}$. Further thermal annealing at $250{ }^{\circ} \mathrm{C}$ initiates cyclodehydrogenation of the intermediate and its planarization giving the separated bisanthene molecules as documented in Figure $7 \mathrm{c}$. We note that a similar approach should also be effective for other halogen substituents 
providing a general approach for halogen/hydrogen on-surface atomically precise exchange.

\section{CONCLUSIONS}

In conclusion, we have shown that application of atomic hydrogen and its combination with well-established annealing procedures provides synthetic routes in the on-surface chemistry approach. Our experiments have demonstrated that by a proper choice of conditions, numerous paths could be followed. We have shown that it is possible to initiate onsurface reactions that may lead to complete modification of synthesized nanostructures, for instance, through demonstrated desulfurization of GNRs. Furthermore, we have demonstrated that atomic hydrogen could be applied for efficient passivation of GNR termination from $\mathrm{C}-\mathrm{Br}$ and $\mathrm{C}$-metal to uniform $\mathrm{C}-\mathrm{H}$, which is essential in tuning of the electronic properties. Moreover, atomic hydrogen could also be used for separation of molecular compounds trapped originally within the organometallic phase, where the species are often hard to distinguish from pure organic counterparts. Our experiments also give perspective for further development of surfaceassisted techniques, for instance, by application of other atomic reagents. Finally, we have also demonstrated that the unwanted on-surface Ullmann-like coupling byproducts, i.e. bromine, could be effectively removed from the surface with atomic hydrogen leaving hydrocarbon polymers intact. This is of great importance for the development of synthetic protocols in cases, where higher temperature annealing is impossible to carry out, e.g., with fragile precursors or in 2-dimensional covalent lattices. The approach based on atomic substitution with great precision could be potentially extended from onsurface experiments, e.g., for powders. This gives an opportunity for much broader applications in various technological areas.

\section{METHODS}

Experimental Details. All experiments were carried out in the Scienta Omicron STM/AFM microscope working at LHe temperature $(5 \mathrm{~K})$ under ultrahigh vacuum conditions $\left(<2 \times 10^{-10} \mathrm{mbar}\right)$. The atomically clean $\mathrm{Au}(111)$ surface was prepared by sequential and repetitive cycles of standard ion bombardment $\left(\mathrm{Ar}^{+}\right)$and further thermal annealing to $450{ }^{\circ} \mathrm{C}$. To obtain high-resolution nc-AFM images, the procedure described by Gross et al. was applied. ${ }^{29}$ Molecular precursors were deposited from a Knudsen cell (Kentax $\mathrm{GmbH})$ by thermal evaporation $\left(T_{\mathrm{DBBA}} \sim 145^{\circ} \mathrm{C}, T_{\mathrm{TBPDT}} \sim 210^{\circ} \mathrm{C}\right)$, where the molecular flux was calibrated with the application of the quartz microbalance (in the range of $0.1 \mathrm{~Hz} / \mathrm{min}$ ). The hydrogenation procedure was performed by using a home-built hydrogen cracker with hot filament (thermal dissociation of the molecular hydrogen, purity $>99.99 \%$, additional information in the SI). The gas line with molecular hydrogen was connected with the hydrogen cracker by the precise leak valve. Before the hydrogenation the sample was placed centrally directly in front of the cracker (angle between cracker and the sample surface was kept at $85^{\circ}$ ) and the temperature was set at the desired value. The temperature was measured by a thermocouple (type $\mathrm{K}$ ) mounted on the manipulator very close to the sample. To clean the surface of reaction byproducts and quench the organometallic state the temperature of the sample was set at 50-100 ${ }^{\circ} \mathrm{C}$. The higher temperature $\left(250-350{ }^{\circ} \mathrm{C}\right)$ was used for substitution of the heteroatoms within the molecular nanostructures. The hydrogen pressure in both cases was maintained at $1 \times 10^{-7} \mathrm{mbar}$. The time of the hydrogenation was set in the range of $10-20 \mathrm{~min}$.

\section{ASSOCIATED CONTENT}

\section{Supporting Information}

The Supporting Information is available free of charge at https://pubs.acs.org/doi/10.1021/acsnano.0c05160.

nc-AFM images of faulty stacked S-doped 5-AGNRS; proposed mechanism for the desulfurization on gold surface with atomic hydrogen; additional information on the hydrogen cracker (PDF)

\section{AUTHOR INFORMATION}

\section{Corresponding Author}

Szymon Godlewski - Centre for Nanometer-Scale Science and Advanced Materials, NANOSAM, Faculty of Physics, Astronomy and Applied Computer Science, Jagiellonian University, PL 30-348 Kraków, Poland; (1) orcid.org/00000002-8515-1566; Email: szymon.godlewski@uj.edu.pl

\section{Authors}

Rafal Zuzak - Centre for Nanometer-Scale Science and Advanced Materials, NANOSAM, Faculty of Physics, Astronomy and Applied Computer Science, Jagiellonian

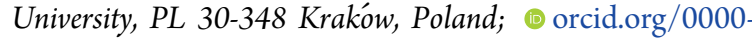
0001-6617-591X

Andrej Jančařík - CNRS, 31055 Toulouse, France; Institute of Organic Chemistry and Biochemistry of the Czech Academy of Sciences, 16610 Prague 6, Czech Republic

Andre Gourdon - CNRS, 31055 Toulouse, France; (1) orcid.org/0000-0002-0370-1019

Marek Szymonski - Centre for Nanometer-Scale Science and Advanced Materials, NANOSAM, Faculty of Physics, Astronomy and Applied Computer Science, Jagiellonian University, PL 30-348 Krakow, Poland

Complete contact information is available at:

https://pubs.acs.org/10.1021/acsnano.0c05160

\section{Author Contributions}

R.Z. performed all STM and nc-AFM measurements. S.G. and R.Z. conceived the original idea. S.G. and M.S. supervised the experiments. A.G. and A.J. synthesized TBPDT precursors. The manuscript was written by S. G. All authors have given approval to the final version of the manuscript.

\section{Notes}

The authors declare no competing financial interest.

\section{ACKNOWLEDGMENTS}

We acknowledge financial support from the National Science Center, Poland (2019/35/B/ST5/02666), and the EU project PAMS (610446). The STM experiments were carried out using equipment purchased with financial support from the European Regional Development Fund within the framework of the Polish Innovation Economy Operational Program (Contract No. POIG.02.01.00-12-023/08). A.J. acknowledges funding from the Foundation EXPERIENTIA and from ERDF/ESF “UOCHB MSCA Mobility” (No. CZ.02.2.69/ 0.0/0.0/17_050/0008490). R.Z. acknowledges support received from the National Science Center, Poland (2017/24/ T/ST5/00262).

\section{REFERENCES}

(1) Clair, S.; de Oteyza, D. G. Controlling a Chemical Coupling Reaction on a Surface: Tools and Strategies for On-Surface Synthesis. Chem. Rev. 2019, 119, 4717-4776. 
(2) Grill, L.; Dyer, M.; Lafferentz, L.; Persson, M.; Peters, M. V.; Hecht, S. Nano-Architectures by Covalent Assembly of Molecular Building Blocks. Nat. Nanotechnol. 2007, 2, 687-691.

(3) Cai, J.; Ruffieux, P.; Jaafar, R.; Bieri, M.; Braun, T.; Blankenburg, S.; Muoth, M.; Seitsonen, A. P.; Saleh, M.; Feng, X.; Müllen, K.; Fasel, R. Atomically Precise Bottom-Up Fabrication of Graphene Nanoribbons. Nature 2010, 466, 470-473.

(4) Ruffieux, P.; Wang, S.; Yang, B.; Sánchez-Sánchez, C.; Liu, J.; Dienel, T.; Talirz, L.; Shinde, P.; Pignedoli, C. A.; Passerone, D.; Dumslaff, T.; Feng, X.; Müllen, K.; Fasel, R. On-Surface Synthesis of Graphene Nanoribbons with Zigzag Edge Topology. Nature 2016, 531, 489-493.

(5) Rogers, C.; Chen, C.; Pedramrazi, Z.; Omrani, A. A.; Tsai, H. Z.; Jung, H. S.; Lin, S.; Crommie, M. F.; Fischer, F. R. Closing the Nanographene Gap: Surface-Assisted Synthesis of Peripentacene from 6,6'-Bipentacene Precursors. Angew. Chem., Int. Ed. 2015, 54, 1514315146

(6) Zuzak, R.; Pozo, I.; Engelund, M.; Garcia-Lekue, A.; Vilas-Varela, M.; Alonso, J. M.; Szymonski, M.; Guitián, E.; Pérez, D.; Godlewski, S.; Peña, D. Synthesis and Reactivity of a Trigonal Porous Nanographene on a Gold Surface. Chem. Sci. 2019, 10, 10143.

(7) Xu, K.; Urgel, J. I.; Eimre, K.; Di Giovannantonio, M.; Keerthi, A.; Komber, H.; Wang, S.; Narita, A.; Berger, R.; Ruffieux, P.; Pignedoli, C. A.; Liu, J.; Müllen, K.; Fasel, R.; Feng, X. On-Surface Synthesis of a Nonplanar Porous Nanographene. J. Am. Chem. Soc. 2019, 141, 7726-7730.

(8) Zuzak, R.; Castro-Esteban, J.; Brandimarte, P.; Engelund, M.; Cobas, A.; Piątkowski, P.; Kolmer, M.; Pérez, D.; Guitián, E.; Szymonski, M.; Sánchez-Portal, D.; Godlewski, S.; Peña, D. Building a 22-Ring Nanographene by Combining In-Solution and On-Surface Syntheses. Chem. Commun. 2018, 54, 10256-10259.

(9) Pavliček, N.; Mistry, A.; Majzik, Z.; Moll, N.; Meyer, G.; Fox, D. J.; Gross, L. Synthesis and Characterization of Triangulene. Nat. Nanotechnol. 2017, 12, 308-311.

(10) Mishra, S.; Beyer, D.; Eimre, K.; Liu, J.; Berger, R.; Gröning, O.; Pignedoli, C. A.; Müllen, K.; Fasel, R.; Feng, X.; Ruffieux, P. Synthesis and Characterization of $\pi$-Extended Triangulene. J. Am. Chem. Soc. 2019, 141, 10621-10625.

(11) Mishra, S.; Beyer, D.; Eimre, K.; Kezilebieke, S.; Berger, R.; Gröning, O.; Pignedoli, C. A.; Müllen, K.; Liljeroth, P.; Ruffieux, P.; Feng, X.; Fasel, R. Topological Frustration Induces Unconventional Magnetism in a Nanographene. Nat. Nanotechnol. 2020, 15, 22-28.

(12) Franc, G.; Gourdon, A. Covalent Networks through On-Surface Chemistry in Ultra-High Vacuum: State-Of-The-Art and Recent Developments. Phys. Chem. Chem. Phys. 2011, 13, 14283-14292.

(13) Lackinger, M. Surface-Assisted Ullmann Coupling. Chem. Commun. 2017, 53, 7872-7885.

(14) Xiao, Z.; Ma, C.; Lu, W.; Huang, J.; Liang, L.; Hong, K.; Li, A.P.; Sumpter, B. G.; Bernholc, J. Ab Initio Investigation of the Cyclodehydrogenation Process for Polyanthrylene Transformation to Graphene Nanoribbons. NPJ. Comput. Mater. 2019, 5, 91.

(15) Roman, T.; Gossenberger, F.; Forster-Tonigold, K.; Gro $\beta$, A. Halide Adsorption on Close-Packed Metal Electrodes. Phys. Chem. Chem. Phys. 2014, 16, 13630-13634.

(16) Basagni, A.; Vasseur, G.; Pignedoli, C. A.; Vilas-Varela, M.; Peña, D.; Nicolas, L.; Vitali, L.; Lobo-Checa, J.; de Oteyza, D. G.; Sedona, F.; Casarin, M.; Ortega, J. E.; Sambi, M. Tunable Band Alignment with Unperturbed Carrier Mobility of On-Surface Synthesized Organic Semiconducting Wires. ACS Nano 2016, 10, 2644-2651.

(17) Vasseur, G.; Fagot-Revurat, Y.; Sicot, M.; Kierren, B.; Moreau, L.; Malterre, D.; Cardenas, L.; Galeotti, G.; Lipton-Duffin, J.; Rosei, F.; Di Giovannantonio, M.; Contini, G.; Le Fèvre, P.; Bertran, F.; Liang, L.; Meunier, V.; Perepichka, D. F. Quasi One-Dimensional Band Dispersion and Surface Metallization in Long-Range Ordered Polymeric Wires. Nat. Commun. 2016, 7, 10235.

(18) Fan, Q.; Gottfried, J. M.; Zhu, J. Surface-Catalyzed C-C Covalent Coupling Strategies toward the Synthesis of Low-Dimen- sional Carbon-Based Nanostructures. Acc. Chem. Res. 2015, 48, 2484-2494.

(19) Eichhorn, J.; Strunskus, T.; Rastgoo-Lahrood, A.; Samanta, D.; Schmittel, M.; Lackinger, M. On-Surface Ullmann Polymerization via Intermediate Organometallic Networks on $\mathrm{Ag}(111)$. Chem. Commun. 2014, 50, 7680-7682.

(20) Dong, L.; Liu, P. N.; Lin, N. Surface-Activated Coupling Reactions Confined on a Surface. Acc. Chem. Res. 2015, 48, 27652774.

(21) Li, J.; Martin, K.; Avarvari, N.; Wäckerlin, C.; Ernst, K.-H. Spontaneous Separation of On-Surface Synthesized Tris-Helicenes into Two-Dimensional Homochiral Domains. Chem. Commun. 2018, 54, 7948-7951.

(22) Mairena, A.; Baljozovic, M.; Kawecki, M.; Grenader, K.; Wienke, M.; Martin, K.; Bernard, L.; Avarvari, N.; Terfort, A.; Ernst, K.-H.; Wäckerlin, C. The Fate of Bromine after Temperature-Induced Dehydrogenation of On-Surface Synthesized Bisheptahelicene. Chem. Sci. 2019, 10, 2998-3004.

(23) Zagranyarski, Y.; Chen, L.; Jansch, D.; Gessner, T.; Li, C.; Müllen, K. Toward Perylene Dyes by the Hundsdiecker Reaction. Org. Lett. 2014, 16, 2814-2817.

(24) Tran, V.; Pham, T. A.; Grunst, M.; Kivala, M.; Stöhr, M. Surface-Confined [2 + 2] Cycloaddition towards One-Dimensional Polymers Featuring Cyclobutadiene Units. Nanoscale 2017, 9, 18305.

(25) Nguyen, M.-T.; Pignedoli, C. A.; Passerone, D. An Ab Initio Insight into the $\mathrm{Cu}(111)$-Mediated Ullmann Reaction. Phys. Chem. Chem. Phys. 2011, 13, 154-160.

(26) Saywell, A.; Greń, W.; Franc, G.; Gourdon, A.; Bouju, X.; Grill, L. Manipulating the Conformation of Single Organometallic Chains on $\mathrm{Au}(111)$. J. Phys. Chem. C 2014, 118, 1719-1728.

(27) Telychko, M.; Su, J.; Gallardo, A.; Gu, Y.; Mendieta-Moreno, J. I.; Qi, D.; Tadich, A.; Song, S.; Lyu, P.; Qiu, Z.; Fang, H.; Joo Koh, M.; Wu, J.; Jelínek, P.; Lu, J. Strain-Induced Isomerization in OneDimensional Metal-Organic Chains. Angew. Chem., Int. Ed. 2019, 58, 18591-18597.

(28) Zheltov, V. V.; Cherkez, V. V.; Andryushechkin, B. V.; Zhidomirov, G. M.; Kierren, B.; Fagot-Revurat, Y.; Malterre, D.; Eltsov, K. N. Structural Paradox in Submonolayer Chlorine Coverage on $\mathrm{Au}(111)$. Phys. Rev. B: Condens. Matter Mater. Phys. 2014, 89, 195425

(29) Gross, L.; Mohn, F.; Moll, N.; Liljeroth, P.; Meyer, G. The Chemical Structure of a Molecule Resolved by Atomic Force Microscopy. Science 2009, 325, 1110-1114.

(30) Kawai, S.; Sadeghi, A.; Okamoto, T.; Mitsui, C.; Pawlak, R.; Meier, T.; Takeya, J.; Goedecker, S.; Meyer, E. Organometallic Bonding in an Ullmann-Type On-Surface Chemical Reaction Studied by High-Resolution Atomic Force Microscopy. Small 2016, 12, 5303-5311.

(31) Zhang, H.; Lin, H.; Sun, K.; Chen, L.; Zagranyarski, Y.; Aghdassi, N.; Duhm, S.; Li, Q.; Zhong, D.; Li, Y.; Müllen, K.; Fuchs, H.; Chi, L. On-Surface Synthesis of Rylene-Type Graphene Nanoribbons. J. Am. Chem. Soc. 2015, 137, 4022-4025.

(32) Kimouche, A.; Ervasti, M. M.; Drost, R.; Halonen, S.; Harju, A.; Joensuu, P. M.; Sainio, J.; Liljeroth, P. Ultra-Narrow Metallic Armchair Graphene Nanoribbons. Nat. Commun. 2015, 6, 10177. 